\title{
Development of Personalized Urination Recognition Technology Using Smart Bands
}

\author{
Sung-Jong Eun ${ }^{1}$, Taeg-Keun Whangbo ${ }^{1}$, Dong Kyun Park ${ }^{2}$, Khae-Hawn Kim ${ }^{3}$ \\ ${ }^{1}$ Department of Computer Science, Gachon University, Seongnam, Korea \\ ${ }^{2}$ Health IT Research Center, Gachon University Gil Medical Center, Gachon University, Incheon, Korea \\ ${ }^{3}$ Department of Urology, Gachon University Gil Medical Center, Gachon University, Incheon, Korea
}

Purpose: This study collected and analyzed activity data sensed through smart bands worn by patients in order to resolve the clinical issues posed by using voiding charts. By developing a smart band-based algorithm for recognizing urination activity in patients, this study aimed to explore the feasibility of urination monitoring systems.

Methods: This study aimed to develop an algorithm that recognizes urination based on a patient's posture and changes in posture. Motion data was obtained from a smart band on the arm. An algorithm that recognizes the 3 stages of urination (forward movement, urination, backward movement) was developed based on data collected from a 3-axis accelerometer and from tilt angle data. Real-time data were acquired from the smart band, and for data corresponding to a certain duration, the absolute value of the signals was calculated and then compared with the set threshold value to determine the occurrence of vibration signals. In feature extraction, the most essential information describing each pattern was identified after analyzing the characteristics of the data. The results of the feature extraction process were sorted using a classifier to detect urination.

Results: An experiment was carried out to assess the performance of the recognition technology proposed in this study. The final accuracy of the algorithm was calculated based on clinical guidelines for urologists. The experiment showed a high average accuracy of $90.4 \%$, proving the robustness of the proposed algorithm.

Conclusions: The proposed urination recognition technology draws on acceleration data and tilt angle data collected via a smart band; these data were then analyzed using a classifier after comparative analyses with standardized feature patterns.

Keywords: Urination Recognition; Urination Monitoring System; Mobile Voiding Chart

- Fund/Grant Support: This research was supported by a grant of the Korea Health Technology R\&D Project through the Korea Health Industry Development Institute (KHIDI), funded by the Ministry of Health \& Welfare, Republic of Korea (grant number: HI16C2319). This work was supported by the Gachon University Gil Medical Center (grant number: 2016-12).

- Research Ethics: This research was approved by Institutional Review Board of Gachon University Gil Medical Center (approval number: GDIRB2017-096).

- Conflict of Interest: KHK, a Associate Editor of INJ, is the corresponding author of this article. However, he played no role whatsoever in the editorial evaluation of this article or the decision to publish it. No potential conflict of interest relevant to this article was reported.

\section{INTRODUCTION}

Hospitals provide various tools to assist in the care of patients with dysuria, such as voiding charts. The voiding chart is de- scribed in textbooks as one of the most important diagnostic tools for dysuria [1-3]. It is a method that allows the doctor to observe the subjective symptoms of the patients in an objective way. Because diagnosis or treatment is carried out after the

Corresponding author: Khae-Hawn Kim (iD http://orcid.org/0000-0002-7045-8004 Department of Urology, Gachon University Gil Medical Center, Gachon University, 21 Namdong-daero 774beon-gil, Namdong-gu, Incheon 21565, Korea

E-mail: kimcho99@gilhospital.com / Tel: +82-32-460-3331 / Fax: +82-32-460-8340

Submitted: March 5, 2017 / Accepted after revision: April 17, 2017 
symptoms are observed objectively, this method is considered the starting point for studies of dysuria. As in the famous saying, "a patient reporting his symptoms is like a witness who cannot be trusted," dysuric symptoms subjectively described by patients can be recounted differently depending on the patient's personality and surroundings, even if the symptoms occur with equal frequency. Unlike history-taking, which is one of the most objective methods used to assess urinary symptoms, the voiding chart is used to make a complete diagnosis based on records collected from the patient's daily activities. In other words, history-taking is used by doctors to observe the urinary symptoms of patients at a certain time, while the voiding chart is used to carry out a more thorough observation. The values of urination frequency or urination volume change may be irregular, showing significant variation according to factors such as body condition, liquid intake, and amount of physical activity. Therefore, the key to diagnosis is quickly identifying statistically meaningful changes in the overall frequency of urination.

However, because such voiding charts are recorded by patients similarly to how journal entries are recorded, they may contain inaccurate data. Even if patients are trained well, as in a clinical study, many studies have shown that the recorded data may be inaccurate [4-6]. In particular, because it is nearly impossible for children and elderly patients to record all variables, these patient groups are generally asked to only record their urination frequency. Moreover, there are many variables that can be evaluated using voiding charts, but it is clinically impossible to apply them to all patients. In other words, patient care is difficult because of the involvement of many variables in voiding charts, which is mainly due to the problem of patients having varying abilities to record their urination patterns themselves.

According to the International Continence Society, voiding charts are used in the following 3 forms [7-10]. A micturition time chart is used to observe the frequency and time distribution of urination over a single day by having children and elderly patients only indicate the time of their urination. A frequency volume chart is used to observe urination activity by having patients indicate not only the time of urination, but also the amount. A bladder diary is used to observe many variables at once, by having patients indicate the time of urination, the amount of urination, the time and amount of urinary incontinence, use of pads, and water intake. The bladder diary is used for patients (e.g., young adults) who are able to record their urination activities well. However, because there are so many vari- ables involved in interpreting these records (largely due to the aforementioned issue of patients having varying abilities to record their activities), it is difficult for such voiding charts to provide accurate measurements of urinary activities. If such issues could be resolved, it would be possible to provide more systemic and efficient care to dysuric patients. Against this backdrop, the current trend is to develop plans to integrate information technology into these recording systems.

This study collected and analyzed activity (movement) data sensed through smart bands worn by patients. Through the development of technology that recognizes urination activity and frequency, this study aimed to examine the feasibility of urination monitoring systems. The urination recognition technology proposed in this study is an extension of pattern recognition technology based on existing signal processors. In general, the most common such services are those that alert users after recognizing an extracted pattern (e.g., a particular motion) in home care services. The proposed technology additionally recognizes the signal pattern of urination, in order to measure the frequency of urination and automatically record data regarding a patient's urinary activities. To examine the feasibility of a urination monitoring system, the accuracy and feasibility of the urination recognition system were assessed. Existing pattern detection technology has mainly been developed in algorithms for the recognition of specific motions, such as falling. A variety of methods are in development, including a recognition technology that uses a 3-axis accelerometer and a tilt sensor to collect and analyze data $[11,12]$, a recognition technology that takes into account an acceleration sensor and impact sounds [13], a recognition technology with a learning method based on neural networks in the image recognition field [14], and methods that develop distribution technology (e.g., support vector machines [SVMs] and hidden Markov models) and projection technology [15]. Research and development into such recognition methods have been in progress for a long time, and the methods have been improved in various ways to resolve inaccuracies in certain situations. Several techniques have been used to make improvements, such as adjusting the technology to best fit a particular application, adjusting the optimal threshold value to enhance accuracy, and adjusting the weighted values. Recently, deep-learning algorithm [16] research has become the trendiest learning method with the most accurate performance. However, the principal limitation of deep-learning technology is that it shows optimal performance only when conditions regarding the adequate quantity and quality of the 
data are satisfied. This study aimed to develop a recognition algorithm suitable for a field in which recognition technology has not yet been applied. To achieve this, the strengths of existing recognition technologies were utilized, and an algorithm recognizing the 3 stages of urination (forward movement, urination, and backward movement) was developed to examine the feasibility of urination monitoring.

\section{MATERIALS AND METHODS}

This study aimed to develop an algorithm that recognizes urination based on the patient's posture or change in posture. The patient's motion data were obtained from a smart band on the arm. An algorithm that recognizes the 3 stages of urination (forward movement, urination, and backward movement) was developed based on data collected from the 3-axis accelerometer and tilt angle data (gyroscope). Greater weight was put on the acceleration data than the tilt angle data in order to provide an automatic calculator of urination frequency. The conceptual diagram used for urination recognition was processed according to the following stages, shown in Fig. 1.

\section{Development of Urination Recognition Technology}

There are 2 main processing stages for pattern recognition using machine learning. First, in the learning stage, pattern char- acteristics are analyzed using data obtained from instances of the behavior to be modeled (for instance, falling), and key information for classifying different patterns is extracted. After completing the learning stage, newly obtained data are classified and recognized. Usually, the learning stage is carried out a single time during the production of the recognition technology, and the recognition stage is carried out every time new data are given. The proposed method used for urination recognition included the following stages, shown in Fig. 2.

\section{Preprocessing}

In the preprocessing stage, the acceleration data and tilt angle data collected from the patient's smart band were converted into a simpler form for analysis. To acquire real-time data from the smart band over a certain period of time (including vibration signals), the absolute value of the signals was calculated and then compared with the set threshold value to determine the occurrence of vibration signals. Recognition may be difficult if the patterns look similar but differ in their location in the time domain during pattern comparison. The issue of mapping signals acquired from different times can be resolved if the signals in the time domain are converted into signals in the frequency domain, since signals with identical characteristics will be located together in the frequency domain.

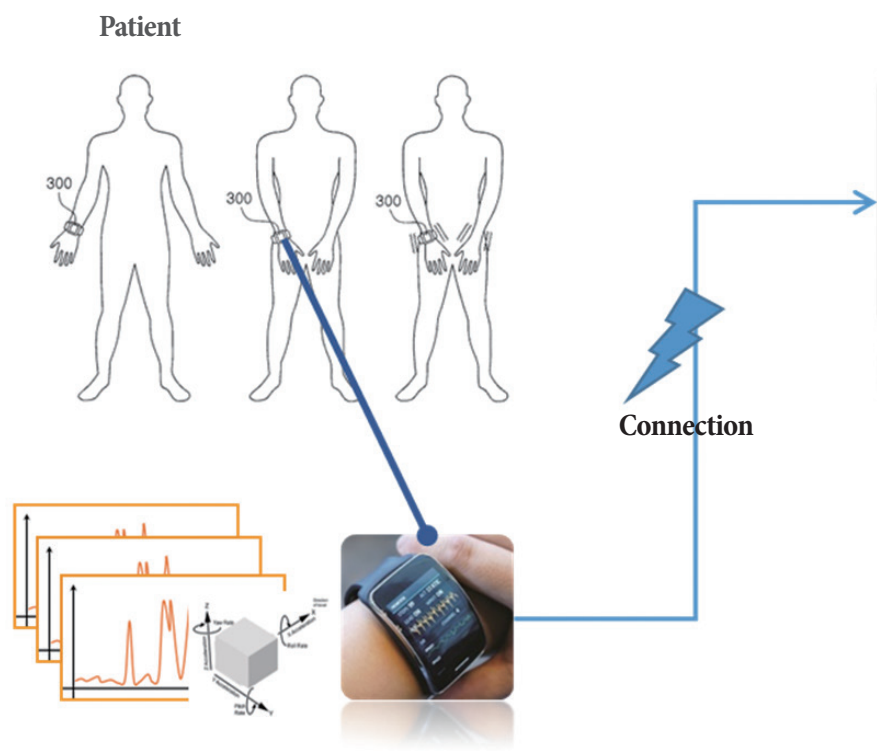

3-axis accelerometer and tilt angle data (gyroscope)
Mobile voiding chart

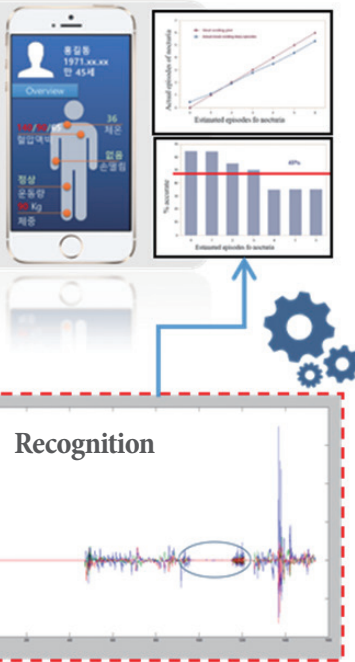

Urination recognition (Proposed method)

Fig. 1. Conceptual diagram of urination recognition. 


\section{Feature extraction process}

During feature extraction, the most essential information that describes each pattern is identified after analyzing the characteristics of the data. Extracting features instead of using the original data can not only save computation and memory, but also eliminate unnecessary information included in the data. First, we confirmed the urination activity features of the accelerometer and tilt angle signal data in the spatial domain. The features of urination activity are different from those of other daily activities. The feature extraction contained 3 stages: forward movement (when the patient prepares for urination), urination, and backward movement (when the patient cleans up after urination). Fig. 3 briefly shows the feature pattern.

Feature information can be used to detect features that allow urination and daily activities to be distinguished even after a specific space conversion, making it ultimately possible to recognize the frequency of urination. The final urination recognition results were provided after taking into account the acceleration data and the tilt angle data of the 3 stages.

\section{Pattern sorting process}

The urination data features extracted from the feature extraction process were sorted using a classifier to detect urination. If similar features are sorted into a feature space, they should be sorted using a nonlinear classifier since the decision field generally has nonlinear characteristics. In this study, patterns were sorted using the k-nearest-neighbors (k-NN) method, a nonlinear classifier of sorts. First, the standard patterns for each

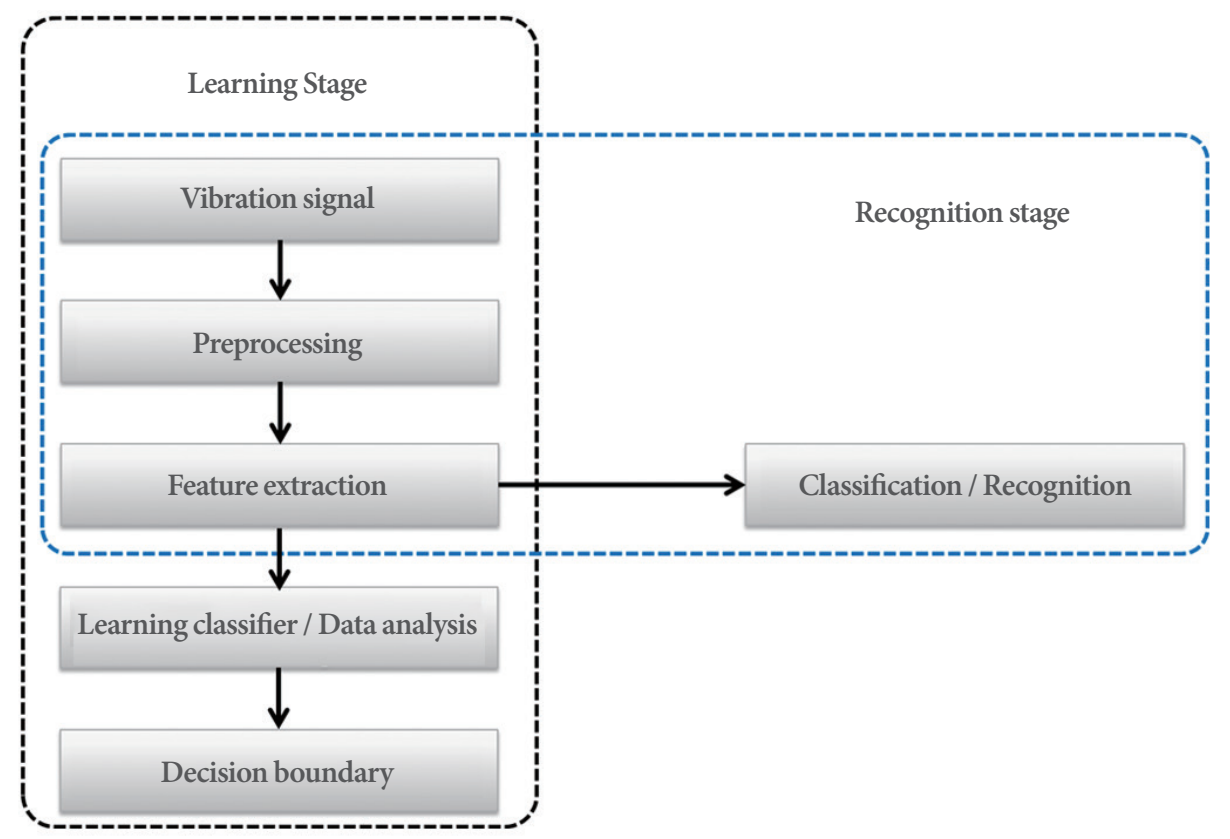

Fig. 2. The complete process of the proposed method for urination recognition.

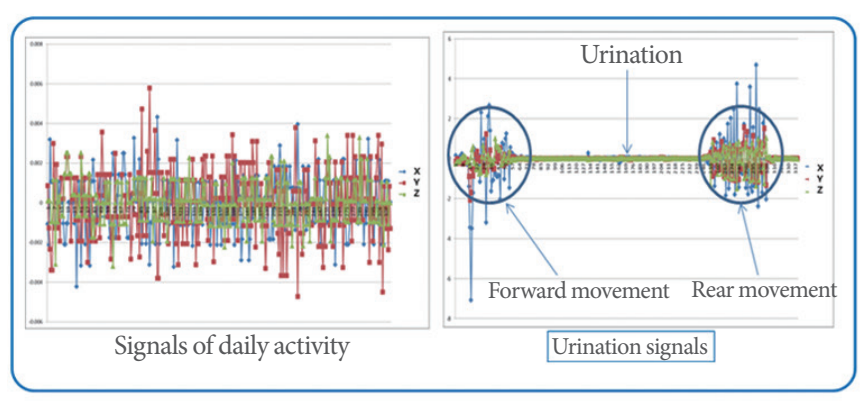

Accelerometer feature sample

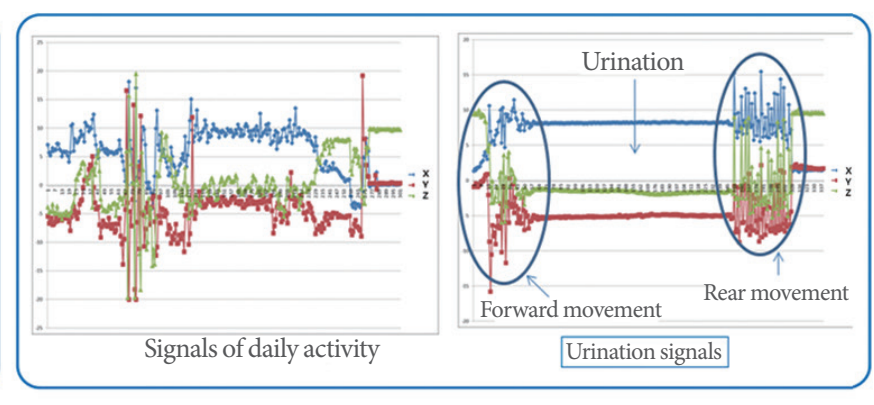

Tilt angle feature sample

Fig. 3. Features of accelerometer and tilt angle information. 
class were saved. A standard pattern was defined as a pattern in which the signal data showing the characteristics of the 3 stages of urination were normalized. After calculating the distance between the saved standard patterns and the signal data, the results were passed onto the final stage, in which urination recognition was performed. The classification rule based on this basic principle is called minimum distance classification. Standard patterns must be selected beforehand for each class for minimum distance classification. The minimum distance classification procedure is as follows. For a class of $\left\{\omega_{i}\right\}_{i=1}^{c}$ and a standard pattern of $\left\{y_{i}\right\}_{i=1}^{m}$, the minimum distance classification rule $\left\{y_{i}\right\}_{i=1}^{m}$ used to classify a random entry pattern $X$ selects the class of the closest xpattern to $X$. In other words, the nearestneighbors (NN) rule is calculated using the following formula:

$$
\left\|X-y_{j}\right\|=\min \left\{\left\|X-y_{j}\right\|\right\}_{j=1}^{m} \Longrightarrow X \in \omega_{j}
$$

The basic minimum distance classification rule given in above formula selects $k$ minimum-distance neighbors of $X$, and can be expanded by applying the majority rule, which is called the k-NN rule. The k-NN classifier based on the k-NN rule can be generated by the pattern classification algorithm as follows:

\section{- Input:}

The difference $\mathrm{k}$ of the NN rule; the entry pattern $X$; the number of standard patterns $m$; the number of pattern classes $C$; the pair $\left(Y_{i}, j_{i}\right)$ of the standard pattern $Y_{i}, 1 \leq i \leq m$, and its class $j_{i}$, $1 \leq j_{i} \leq m$.

\section{- Output:}

The class number $I$, to which the entry pattern belongs.

(1) After carrying out $S \longleftarrow\left\{\left(y_{i}, j_{i}\right)\right\}_{i=1}^{m}$, find $\left(y, j_{0}\right)$ that satisfies

$$
\|y-X\|=\min \left\{\left\|y_{i}-X\right\|\right\}_{i=1}^{m},(y, j) \in S .
$$

(2) If $k=1$, end after $I=j_{0}$. If not, reset to $I C\left(i^{1}\right) \longleftarrow 0, i^{1} \neq j_{0}$; IC $\left(j_{0}\right) \longleftarrow 1$ with the $c$ dimension vector and carry out $S \leftarrow S-\left\{\left(y, j_{0}\right)\right\}$.

(3) Repeatedly carry out the next steps $k-1$ times.

(a) Find $\left(y_{1}, j_{0}\right)$ that satisfies the

$$
\|y-X\|=\min \left\{\left\|y_{i}-X\right\|\right\}_{i=1}^{m},(y, j) \in S .
$$

(b) Carry out $I C\left(j_{0}\right) \longleftarrow I C\left(j_{0}\right)+1, S \longleftarrow S-\left\{\left(y, j_{0}\right)\right\}$

(4) End after $I \leftarrow \operatorname{argmax}\{I C(i)\}_{i=1}^{c}$.

\section{Calculating final pattern similarity}

In this stage, the final recognition rates of the acceleration data and tilt angle data were calculated. Similarities within the accelerometer data were calculated and adjusted using a weighted value of 0.65 , and the final tilt angle was calculated with a weighted value of 0.35 . Compared to the clinical guidelines, the weighted values that exhibited the most optimal recognition rate were calculated experimentally. Generally, the importance of the acceleration data was somewhat higher for optimizing accurate urination recognition. The following algorithm shows the process through which the final urination recognition likelihood was calculated by assigning weighted values to the acceleration data and tilt angle data:

$$
\sum_{s=1}^{3}\left\{\left(1-D 1_{s}\right) \times \alpha_{1}+\left(1-D 2_{s}\right) \times \alpha_{2}\right\},
$$

where $S$ is 1, 2, $3(=\mathrm{x}, \mathrm{y}, \mathrm{z})$ in the 3-axis (acceleration, tilt angle) data obtained from signals throughout the day, $D 1$ and $D 2$ correspond to the distance based on the acceleration data and tilt angle data, and $\alpha_{1}, \alpha_{2}$ are weighted values as explained above. Based on the results derived from the above algorithm, if the final pattern similarity was greater than $90 \%$, it was determined that urinary activity was present. The frequency and time of the instances of urination were then derived.

\section{RESULTS}

\section{Preprocessing and feature extraction}

To acquire real-time data from the smart band over a certain period of time (including vibration signals), the absolute value of the signals was calculated and then compared with the set threshold value to determine the occurrence of vibration signals. The process and results of this conversion are shown in Fig. 4.

\section{Pattern sorting and recognition}

The feature extraction process involved 3 stages: forward movement (when the patient prepares for urination), urination, and backward movement (when the patient cleans up after urination). The final urination recognition results were obtained after taking into account the acceleration data and the tilt angle data of the 3 stages. Fig. 5 shows a sample of the final recognition signals detected from pattern classification. 


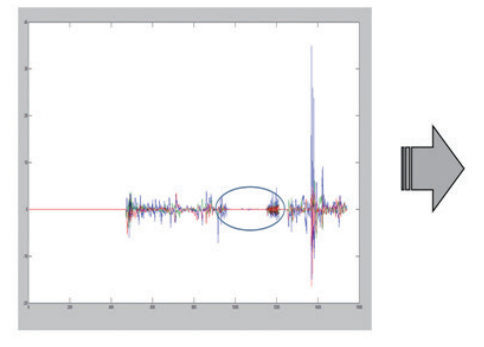

Sample of acceleration data (3-axis)

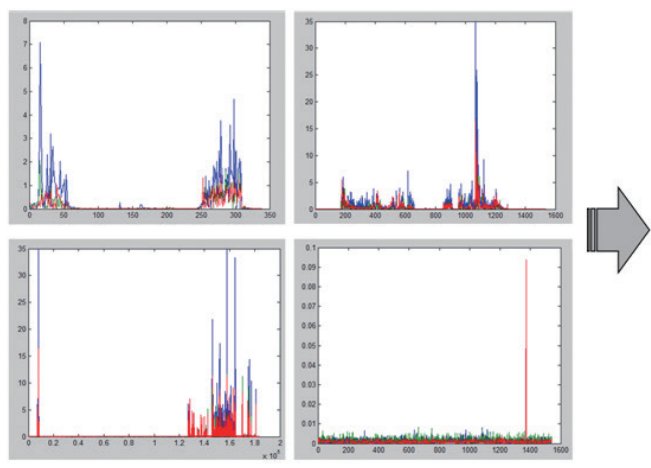

Result of preprocessing

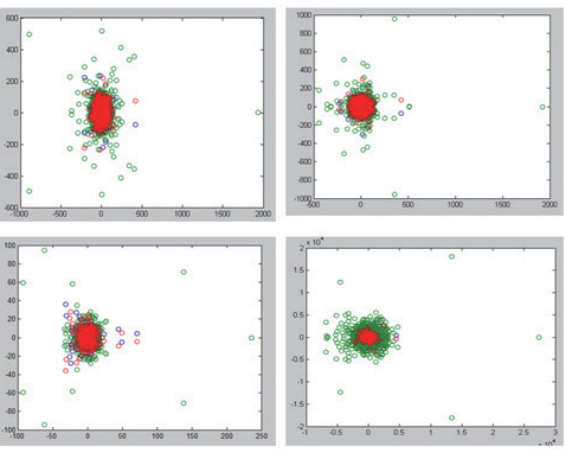

Transformation for feature extraction

Fig. 4. The steps of preprocessing and feature extraction.

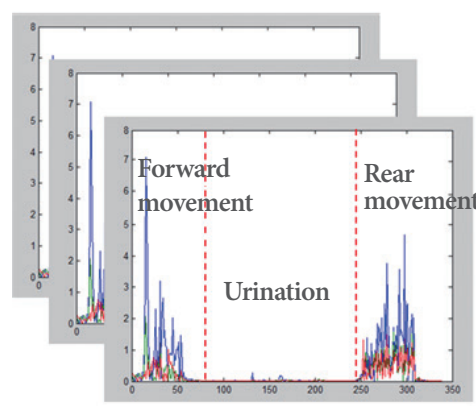

Learning \& pattern sorting

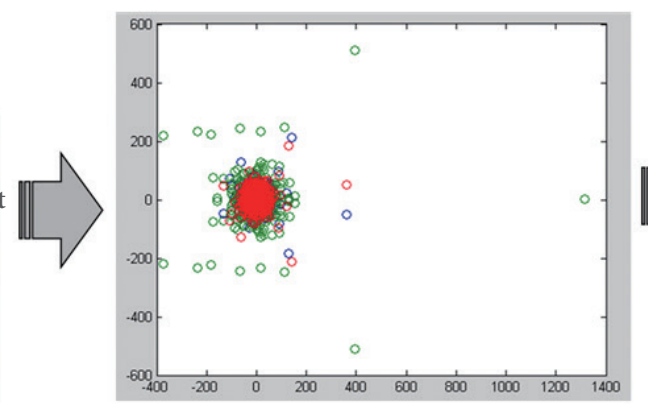

Standard pattern

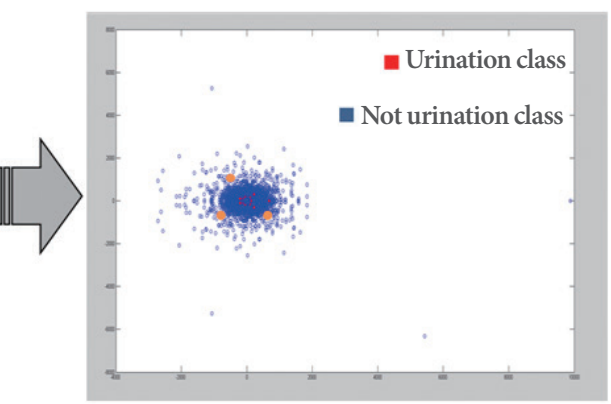

Decision by using k-NN

Fig. 5. The steps of pattern sorting and recognition. k-NN, k-nearest-neighbors.

Table 1. Results of comparison of the proposed method with the other exist methods

\begin{tabular}{lccc}
\hline $\begin{array}{c}\text { Confusion } \\
\text { matrix }\end{array}$ & $\begin{array}{c}\text { Proposed } \\
\text { method }\end{array}$ & $\begin{array}{c}\text { Threshold- } \\
\text { based [12] }\end{array}$ & $\begin{array}{c}\text { SVM- } \\
\text { based [15] }\end{array}$ \\
\hline True positive & 45 & 21 & 40 \\
False positive & 5 & 29 & 10 \\
True negative & 45 & 41 & 41 \\
False negative & 5 & 9 & 9 \\
\hline
\end{tabular}

SVM, support vector machine.

\section{Experiment}

An experiment was carried out to assess the performance of the recognition technology proposed in this study. A total of $20 \mathrm{pa}-$ tients were selected, and the performance of the algorithm was assessed based on the data (acceleration, tilt angle) collected from smart bands for 50 days. The final accuracy of the algorithm was calculated based on voiding charts verified by urologists. The comparative evaluation of the proposed method utilized the threshold method [12] and the commonly used SVM method [15]. This comparative evaluation was performed in order to ensure that the classification was as usable as possible.
We used a confusion matrix for this experiment, in which the accuracy, sensitivity, and specificity of the suggested algorithm and the typical algorithm were compared. True positive (TP), false positive (FP), false negative (FN), and true negative (TN) results were defined as follows:

TP: A match for urination is determined to correctly reflect a real urination event.

FP: A match for urination is determined not to reflect a real urination event.

$\mathrm{TN}$ : A nonmatch for urination is determined to correctly reflect the absence of urination.

FN: A non-match for urination is determined to correspond to a real urination event.

The confusion matrix was calculated, and the average results are shown below (Table 1). Based on the confusion matrix, accuracy, sensitivity, and specificity can be calculated as follows (Fig. 6):

$$
\begin{aligned}
& \text { Accuracy }=(T N+T P) /(T N+T P+F N+F P) \\
& \text { Sensitivity }=T P /(T P+F N) \\
& \text { Specificity }=T N /(T N+F P)
\end{aligned}
$$




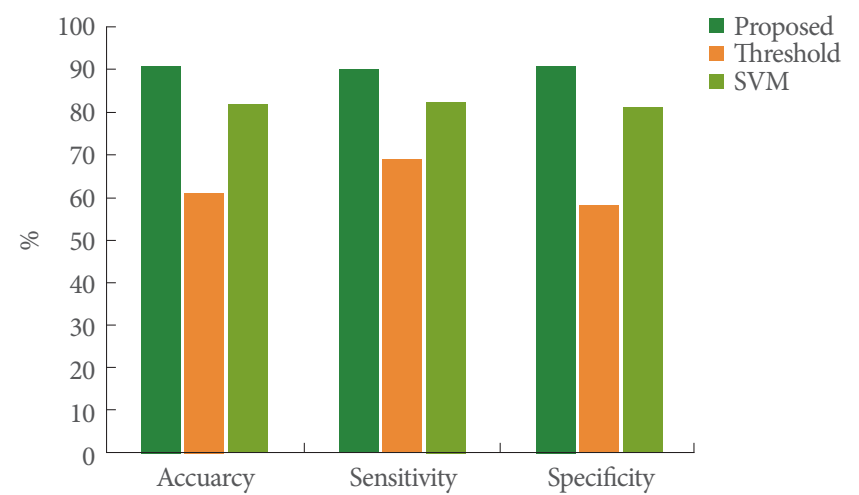

Fig. 6. Results of the overall analysis. SVM, support vector machine.

The calculated confusion matrix and the accuracy, sensitivity, and specificity results confirmed that the suggested method was more accurate than the traditional algorithms. The suggested method was further confirmed to be more effective with a receiver operating characteristic (ROC) curve. ROC curves are often used in medical science and dynamics. An ROC curve is drawn based on the average value of the sensitivity and specificity of the measured values. As shown in Fig. 7, an area under the curve (AUC) of 1 would represent perfect results. If the AUC is 0.5 or less, the method is not useful. AUC values of $0.9-$ 1 represent very accurate results, and values of $0.7-0.89$ represent comparatively accurate results. The ROC curve method further confirmed that our algorithm led to more accurate results than the existing comparison methods.

The experiment showed a high average accuracy rate of $90.4 \%$, proving the robustness of the proposed algorithm. In fact, the threshold method should be applied only to specific criteria, and incorrect results were derived when the individual differences were large. For SVM, inaccurate results were derived with respect to the values of features in the vicinity of the boundary line, and the SVM method has the limitation of requiring a 3-dimensional method to be used. Overall, the results were highly accurate during the morning, afternoon, and evening, but they were less accurate during the early hours for some patients with less movement (average, $72.4 \%$ ). This issue seems to arise from the similarity in the motionless section during the stage of urination recognition to movements during sleep. To resolve this issue, 2 methods can be considered: a higher weighted value could be given for the tilt angle data regarding urination recognition during sleep by identifying the time on the smart band, or a deep-learning method based on a

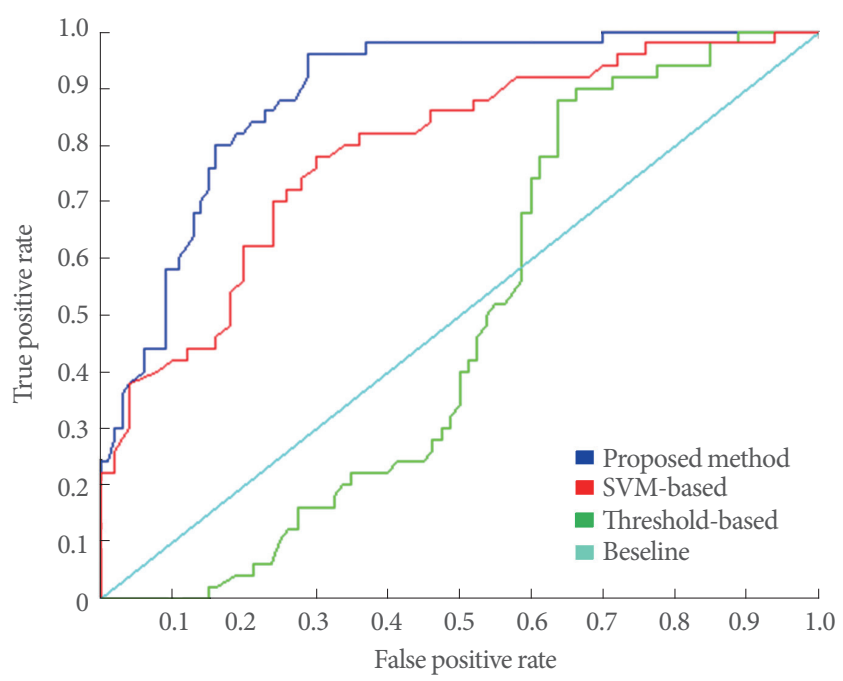

Fig. 7. Receiver operating curve of the overall analysis. SVM, support vector machine.

recurrent neural network (RNN) could be used after the accumulation of sufficient data.

\section{DISCUSSION}

This study developed and applied an automatic urination recognition algorithm to examine the feasibility of developing an automated urination management system for male patients with dysuria. The process of the proposed urination recognition technology began with the collection of acceleration data and tilt angle data through smart bands, which were then analyzed using the k-NN algorithm, a machine-learning method that derives recognition results after comparative analyses with standardized feature patterns. The final optimal recognition results were derived by assigning different weighted values to the results of feature similarity between each set of accelerometer data and the results of feature similarity between each set of tilt angle data. An experiment was conducted to evaluate the performance of this algorithm, targeting actual patients with dysuria. The performance evaluation was carried out based on test data collected over a 50-day period from 20 patients. Since an average accuracy of $90.4 \%$ was obtained, the feasibility of urination recognition was positive. However, while the results were highly accurate during daily activities, they were less accurate during sleeping hours, due to the similarity between the urination stage of the processing model and periods with less movement during sleep. To resolve this issue, 2 methods will be ex- 
plored in the future: a higher weighted value could be given for the tilt angle data during sleeping hours, or a general algorithm could be developed to manage the urination of patients at all times, taking into account a deep-learning method based on an RNN. In addition, the subjects of this study were limited to males only, and in the future, we will explore mechanisms of urination recognition for female patients.

\section{REFERENCES}

1. Klevmark B. Natural pressure-volume curves and conventional cystometry. Scand J Urol Nephrol Suppl 1999;201:1-4.

2. McGuire EJ, Cespedes RD, Cross CA, O'Connell HE. Videourodynamic studies. Urol Clin North Am 1996;23:309-21.

3. Abrams P, Cardozo L, Fall M, Griffiths D, Rosier P, Ulmsten U, et al. The standardisation of terminology of lower urinary tract function: report from the Standardisation Sub-committee of the International Continence Society. Neurourol Urodyn 2002;21:167-78.

4. Jarvis GJ, Hall S, Stamp S, Millar DR, Johnson A. An assessment of urodynamic examination in incontinent women. Br J Obstet Gynaecol 1980;87:893-6.

5. Webb RJ, Fawcett PR, Neal DE. Electromyographic abnormalities in the urethral and anal sphincters of women with idiopathic retention of urine. Br J Urol 1992;70:22-5.

6. Kim SH, Oh SA, Oh SJ. Voiding diary might serve as a useful tool to understand differences between bladder pain syndrome/interstitial cystitis and overactive bladder. Int J Urol 2014;21:179-83.

7. Ku JH, Jeong IG, Lim DJ, Byun SS, Paick JS, Oh SJ. Voiding diary for the evaluation of urinary incontinence and lower urinary tract symptoms: prospective assessment of patient compliance and bur- den. Neurourol Urodyn 2004;23:331-5.

8. Burgio KL, Locher JL, Goode PS, Hardin JM, McDowell BJ, Dombrowski $\mathrm{M}$, et al. Behavioral vs drug treatment for urge urinary incontinence in older women: a randomized controlled trial. JAMA 1998;280:1995-2000.

9. Kim SE, Shin MS, Kim CJ, Park JH, Chung KJ, Jung H, et al. Effects of Tamsulosin on Urinary Bladder Function and Neuronal Activity in the Voiding Centers of Rats with Cyclophosphamide-induced Overactive Bladder. Int Neurourol J 2012;16:13-22.

10. Noh JY, Han DH, Yoon JA, Kim MH, Kim SE, Ko IG, et al. Circadian rhythms in urinary functions: possible roles of circadian clocks? Int Neurourol J 2011;15:64-73.

11. Jung DJ, Shin KW, Kang MH, Cho JM. A study on the development of a long-term data collection device to establish fall detection algorithms, In: 2014 Institute of Electronics and Information Engineers Fall Meeting; 2014 Nov 29; Seoul, Korea. Seoul: The Institute of Electronics and Information Engineers; 2014:11;600-3.

12. Ryu JT. The development of fall detection system using 3-axis acceleration sensor and tilt sensor. J Korea Industr Inf Syst Res 2013: 18:19-23.

13. Kim N. An efficient methodology of fall detection for ubiquitous healthcare, Korean Inst Inf Tech 2010:8:133-40

14. Kim SS, Kim SW, Choi YS. Fall detection system using motion vector. J Korea Inst Inf Electron Commun Tech 2016:9:38-44

15. Ben-Hur A, Ong CS, Sonnenburg S, Schölkopf B, Rätsch G. Support vector machines and kernels for computational biology. PLoS Comput Biolo. 2008;4:e10000173.

16. Lee C. Image caption generation using recurrent neural network. J KIISE 2016:43:878-82. 